\title{
PNEUMATIC PRESSURE CONTROL WITH PUMP FOR NON-INVASIVE BLOOD PRESSURE MEASUREMENT
}

\author{
Toshinori FUJITA* and Toshiharu KAGAWA* \\ * Precision and Intelligence Laboratory \\ Tokyo Institute of Technology \\ 4259 Nagatsuda, Midori-ku, Yokohama, 226-8503 Japan \\ (E-mail: fujita@pms.titech.ac.jp)
}

\begin{abstract}
For the health care the blood pressure has often measured in a home. Until now the blood pressure has been measured when decreasing the cuff pressure, after the cuff is pressurized by the pump. A measuring time is shortened, if the blood pressure is measured in pressurizing the cuff, and the restriction or valve to decrease the cuff pressure is unnecessary. In this study, it was proposed that the blood pressure is measured in the decompression, and for development of this system it discussed some the problems and control methods. As the result, it has been possible that the cuff pressure is risen at the constant pressure speed by only controlling the input voltage of the pump. By applying this, the more human friendly sphygmomanometer is realized.
\end{abstract}

\section{KEY WORDS}

Pneumatics, Blood Pressure Measurement, Pressure Control, Pump, Cuff

\section{NOMENCLATURE}

$\begin{array}{ccll}C m p & : & \text { compliance of cuff } & {\left[\mathrm{m}^{3} / \mathrm{Pa}\right]} \\ K_{p} & : & \text { proportional control gain } & {[\mathrm{V} / \mathrm{kPa}]} \\ P_{c} & : & \text { cuff pressure } & {[\mathrm{kPa}]} \\ \Delta P_{c} & : & \text { pressure change of cuff } & {[\mathrm{s}]} \\ Q & : & \text { flow rate } & {[\mathrm{NL} / \mathrm{min}]} \\ t & : & \text { time } & {[\mathrm{s}]} \\ u & : & \text { input voltage } & {[\mathrm{V}]} \\ \Delta V_{c} & : & \text { volume change of cuff } & {\left[\mathrm{m}^{3}\right]} \\ X & : & \text { length of arm periphery } & {[\mathrm{m}]}\end{array}$

\section{INTRODUCTION}

The blood pressure measurement is very important for the fundamental diagnosis of the circulatory system [1]. Recently the measurement has daily been carried out individually for the health care, and a sphygmomanometer is required that the blood pressure measurement is conveniently and quickly possible. The principle of the non-invasive blood pressure measurement and a example of measured result are shown in Fig. 1 and 2 respectively. The cross section of the artery changes with the change of blood pressure. This change the cuff volume and appears the pressure change in the cuff. The blood pressure is calculated from the profile of pressure change by the oscillograph metric method. Generally, the blood pressure has been measured decreasing the cuff pressure.

Since the characteristic of the cuff is greatly different 
by the thickness of the arm and the cuff pressure, the human with the thick arm needs a long measuring time, and the pressure drop speed slows down as the cuff pressure is lower. In order to solve this problem, authors made the electro-pneumatic proportional valve of the nozzle-flapper type which can adjust the discharging flow, and have been developed the blood pressure measurement system applying this valve and feed back control [2] [3]. As a result, the time of the blood pressure measurement can be constant even in what kind of human is able to be realized. The sphygmomanometer becomes friendly to human. Recently, however it is desired to improve to more friendly system that achieves shortening the time pressurized by the pump shown in Fig.2.

In this paper the new blood pressure measurement
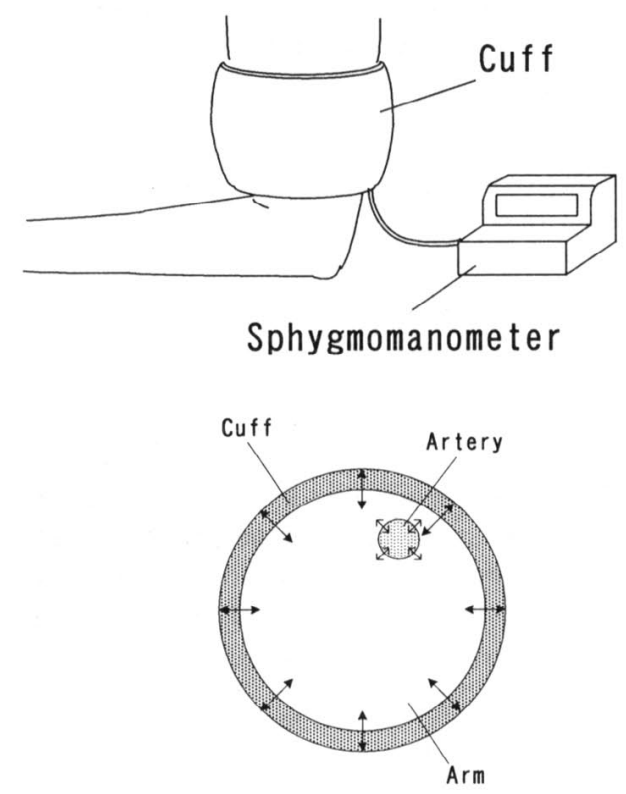

Figure 1 The principle of the non-invasive blood pressure measurement

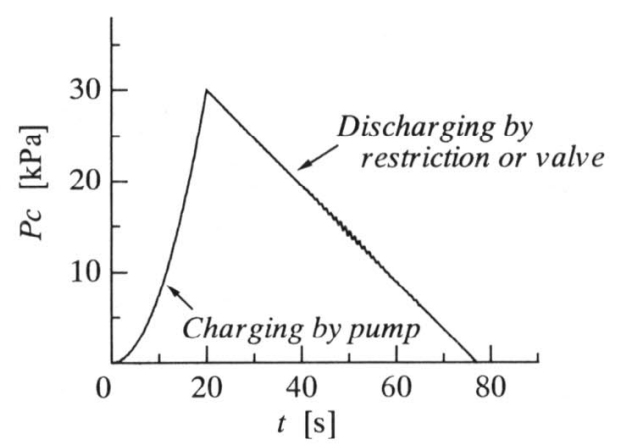

Figure 2 Example of measured result system shown Fig.3 in which the blood pressure is measured in compressing process and appropriate pressurizing speed of the cuff is obtained by controlling the pump. This system can be achieved not only shortening the blood pressure measurement time but size down and cost reduction of the sphygmomanometer, since this method is not need the time for the pressurization and the discharging valve. For the development of this system, characteristics of each element of this system and its control method are discussed.

\section{CHARACTERISTICS OF PUMP AND CUFF}

In this section, characteristics of the pump and the cuff, which are important of the blood pressure measurement system, are described. The pump of Photo. 1 is the piston type with two cylinders and is driven by the DC motor with input of 5[V] maximum voltage. Characteristics of this motor are measured using the equipment of Fig.4. Result are shown in Fig.5. Each line is obtained, when the load pressure is changed by the restriction in making the voltage to be being constant. The linear drooping characteristic has been obtained. However, it is nonlinear characteristic for the voltage in which flow gain in low input voltage is smaller than one in high input voltage. In the voltage of $1.5[\mathrm{~V}]$ or less, the pump can not be driven. This characteristic can be modeled using the equation of the following.

$$
Q=f\left(P_{c}, u\right)
$$

The characteristic of the cuff is important because it governs the speed of the pressure change. The speed of the pressure change is decided by the volume change of the cuff and charging flow rate of the air. The measurement of the volume change is difficult. Therefore, the compliance of the cuff is defined in the following equation, and it is possible to express characteristics of the cuff by this equation.

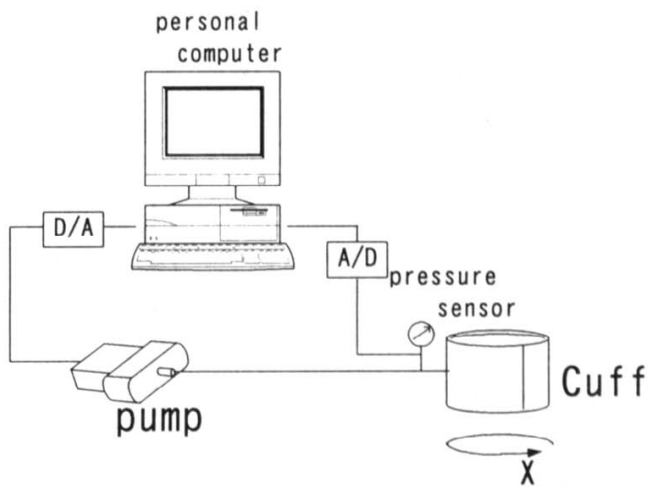

Figure 3 Proposed blood pressure measurement system 


$$
C m p=\frac{\Delta V_{c}}{\Delta P_{c}}
$$

This equation means that the pressure change decreases as the compliance is bigger, when the same quantity of air is charged. This compliance is measured using the apparatus of Fig.6. The compliance is calculated from the pressure change and the quantity of the air sent by the cylinder using Eq.(2). The result is shown in Fig. 7.

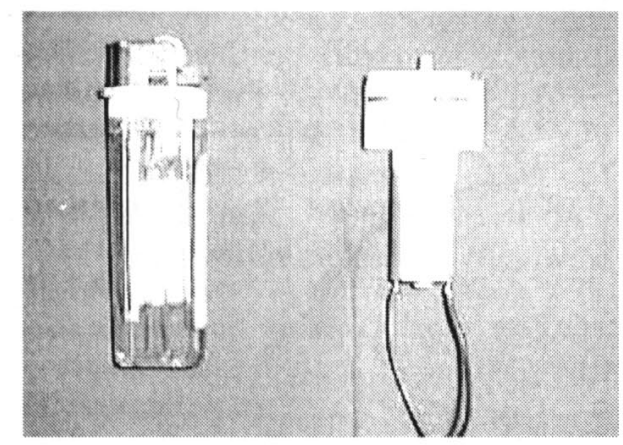

Photograph 1 Charging pump

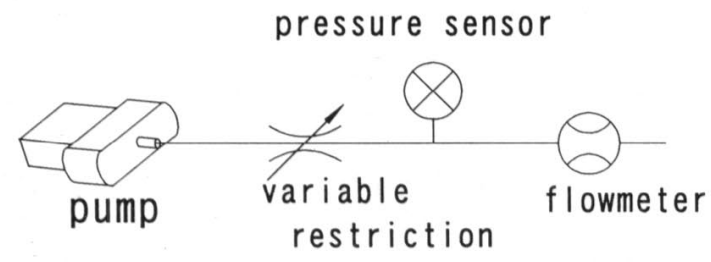

Figure 4 Apparatus for measurement of pump characteristics

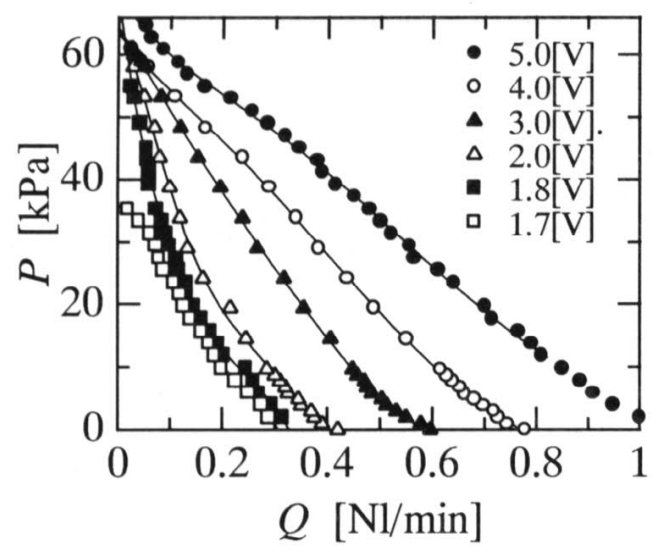

Figure 5 P-Q characteristics of pump
The relation between compliance and pressure is inverse proportion, and large nonlinear characteristic which the compliance increases steeply as the pressure lowers is obtained. It is also found that a compliance is bigger, as the roll is looser, or as the arm is thicker.

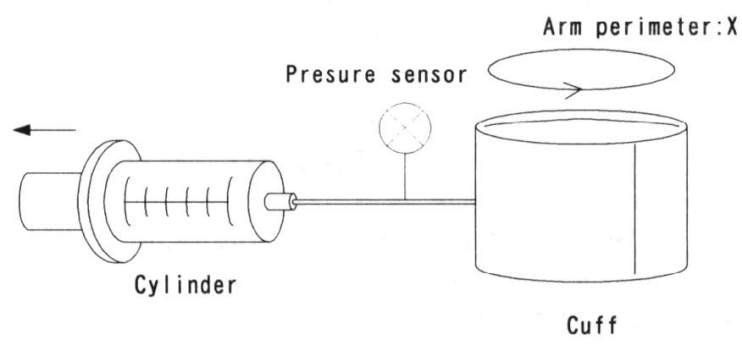

Figure 6 Apparatus for measurement of cuff characteristics

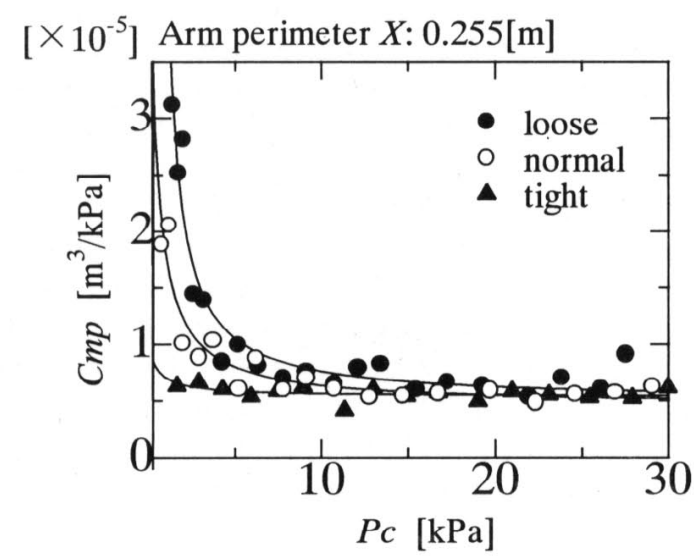

a) Difference by handedness

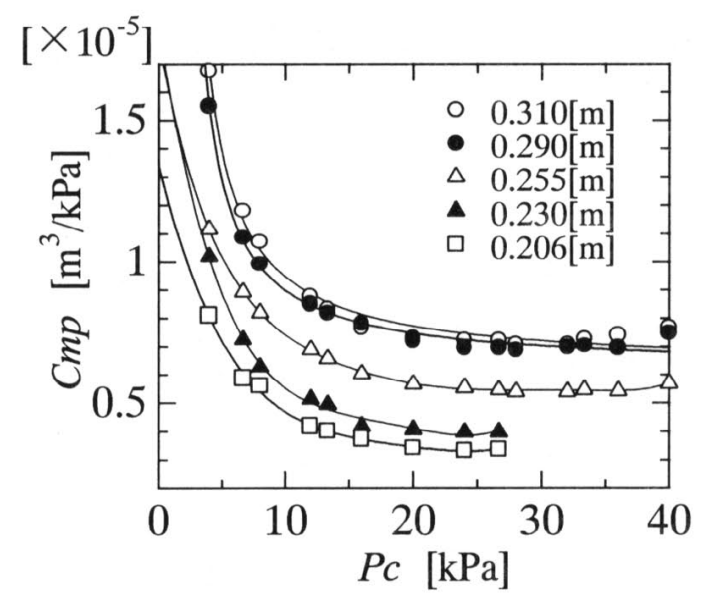

b) Difference by thickness of arm

Figure 7 Compliance characteristics of cuff 
Fig. 8 is the measured result of the cuff pressure in the case of driving the pump by the constant voltage. From this figure, it is necessary that the initial input voltage of the pump should be big in order to obtain equal pressure rise speed in low pressure region and they are reversed in the high pressure region. In addition, since the compliance also changes in proportion to the human, the feedback control in which the pump input automatically changes is needed.

\section{CONTROL SYSTEM FOR BLOOOD PRESSURE MEASUREMENT}

The system is composed of pump, cuff, pressure sensor, microcomputer as shown Fig.3. The pressure must be made to rise at the constant speed for the accurate blood pressure measurement. Then, we control the input voltage to the pump by the microcomputer using the measured cuff pressure. Semi-conductor type pressure sensor is used for the measurement of the cuff pressure.

Observing the pressure response, the pulsation on the curve of the pressure is generated by the pump. Therefore, The amplitude of this pressure pulsation and its frequency are measured. Fig.9 is this result and shown that the frequency is higher and the pressure amplitude decreases being proportional to the input voltage. The frequency is high with over $100[\mathrm{~Hz}]$ and the amplitude of pulsation is over $2[\mathrm{kPa}]$ without relating to the input voltage. This value is bigger than the amplitude of the blood pressure. The remarkable effect is anticipated. Then, the analogue pre-filter made up electrical circuit of Fig.10 a) is installed for treatment of the pressure sensor output. The measured pressure pulsation also include the component caused the blood pressure to should be measured. The fundamental frequency of a blood pressure wave is $1[\mathrm{~Hz}]$ and harmonic under $10[\mathrm{~Hz}]$ are contained in this wave. From the above fact, the filter property was decided in lowpass filter with characteristics shown Fig.10 b). The effect of the filter can be confirmed in Fig.9.

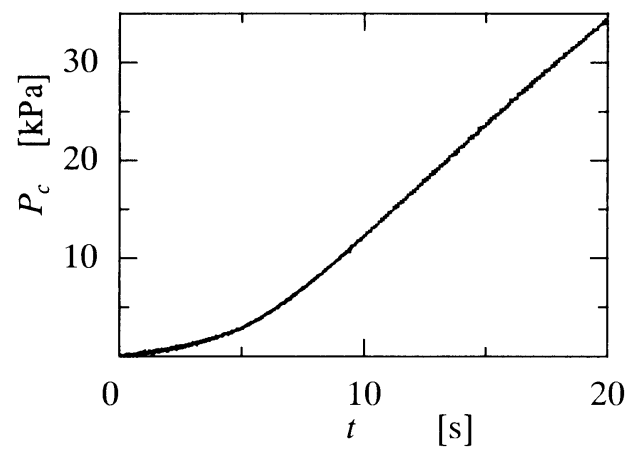

Figure 8 Pressure response of cuff

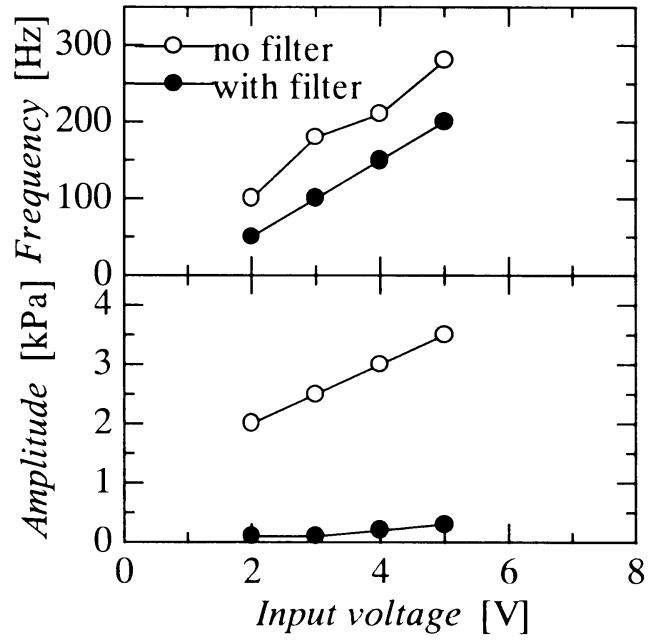

Figure 9 Amplitude of pressure pulsation and its frequency

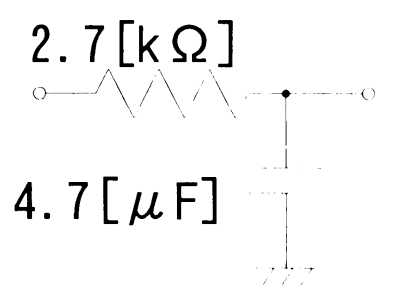

a) Electrical circuit

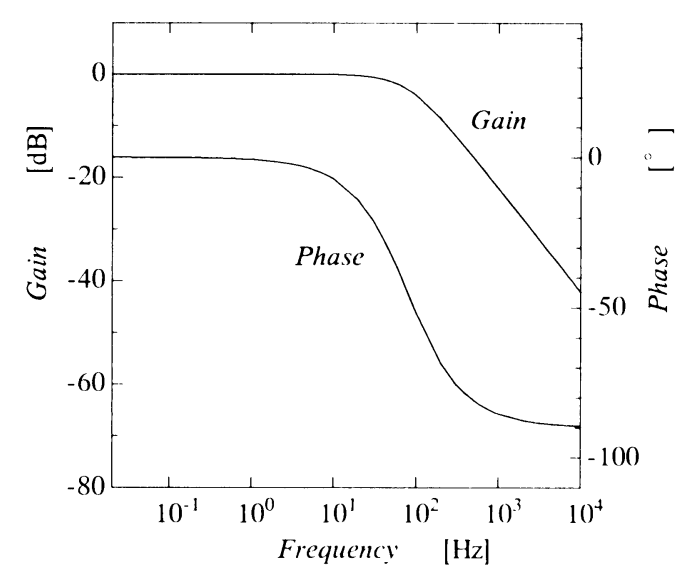

b) Characteristics

Figure 10 Pre-filter 
Fig. 11 is the block diagram describing the blood pressure measurement system. In the microcomputer, the pressure control is also carried out with the blood pressure measurement. The $12 \mathrm{bit} \mathrm{AD}$ converter and the 16 bit DA converter is used on the input and the output to the microcomputer. The sampling time is $20[\mathrm{~ms}]$. The control algorithm is adopted the PI control. The pulsation of the blood pressure, which added to the cuff pressure, is the disturbance for the control system. Reversely in the view of the blood pressure measurement, the pulse wave of the blood pressure is distorted by the control because of the effectiveness suppressing disturbances. Therefore, the lowpass filter, which cut-off frequency is $0.4[\mathrm{~Hz}]$ and damping factor is 1.0 , is also set in the software of the control algorithm.

Fig. 12 is the frequency response from the pressure fluctuation to the measured pressure. It is found that in the region from $1[\mathrm{~Hz}]$ to $10[\mathrm{~Hz}]$ in which the blood pressure component is included, the characteristics is same with the original characteristics of the cuff, that is integral characteristics, and that under $0.5[\mathrm{~Hz}]$ disturbance is suppressed by the control. Fig. 12 proves that the control is not effect to the blood pressure measurement.

\section{RESULTS OF PRESSURE CONTROL}

The control parameter is decided from experimental result designed based on simulation result calculated from equations modeled the control system like Eq.(1) and (2) and root locus method, e.g. Fig.13 or 14. Example of result of controlling the cuff pressure by this system is shown Fig.15. Generally human minimum blood pressure is about $5[\mathrm{kPa}]$. Therefore, the pump is driven to about $4.5[\mathrm{kPa}]$ at the maximum voltage in order to get highest pressurizing speed, and microcomputer switches to the PI control algorithm afterwards. The initial voltage to the pump in the switching PI Control is given the value calculated from the compliance estimated from $3.5[\mathrm{kPa}$ ] to $4.5[\mathrm{kPa}]$

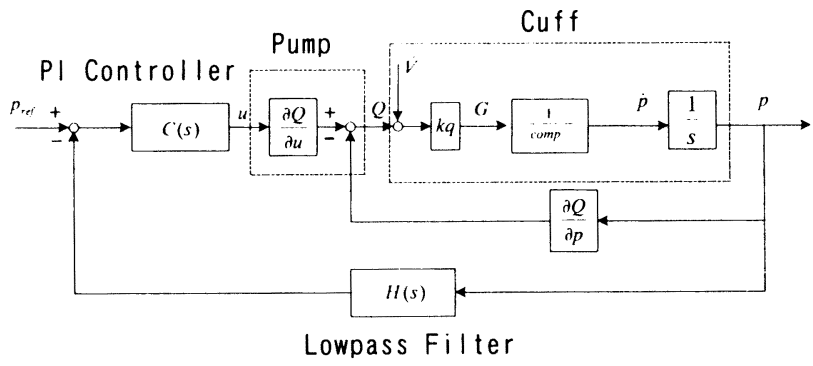

Figure 11 Block diagram of system pressure rise speed. These are devices in the control algorithm. In Fig. 15 a) the pressure rises at the constant speed decided reference without the effect of pressure pulsation of the pump and the blood pressure. In Fig. 15 b) is the measured pressure variation of the cuff, and this result is sufficient as a measurement data of blood pressure.

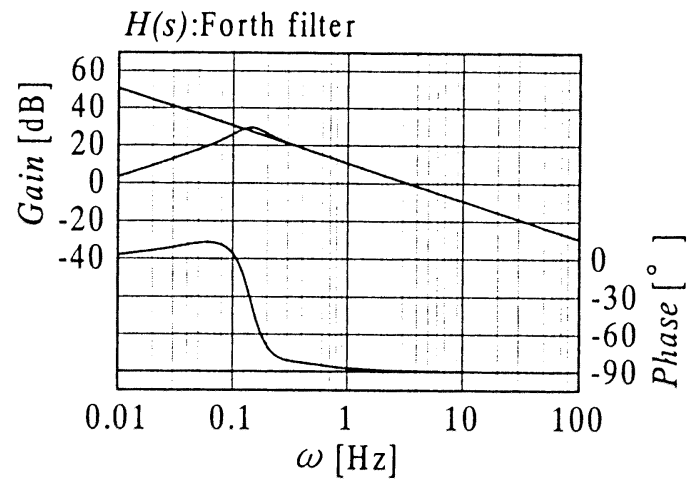

Figure 12 Frequency response from the pressure fluctuation to the measured pressure

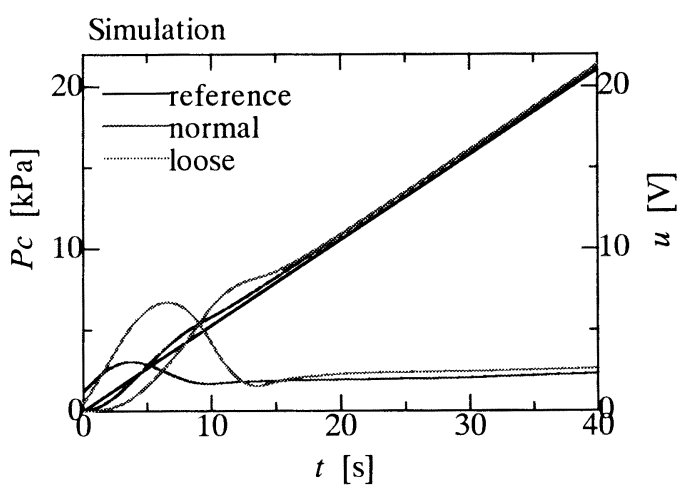

Figure 13 Simulation results evaluated the stability

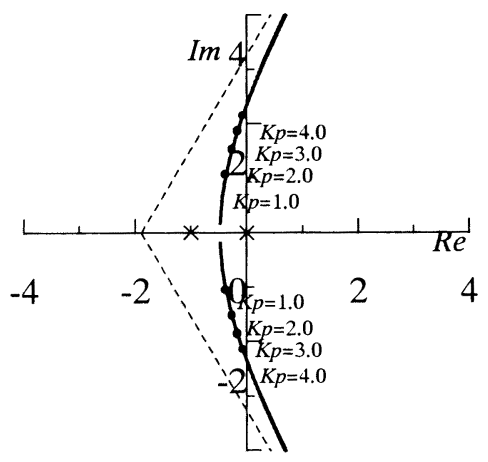

$-4$

Figure 14 Root locus of pressure control system 


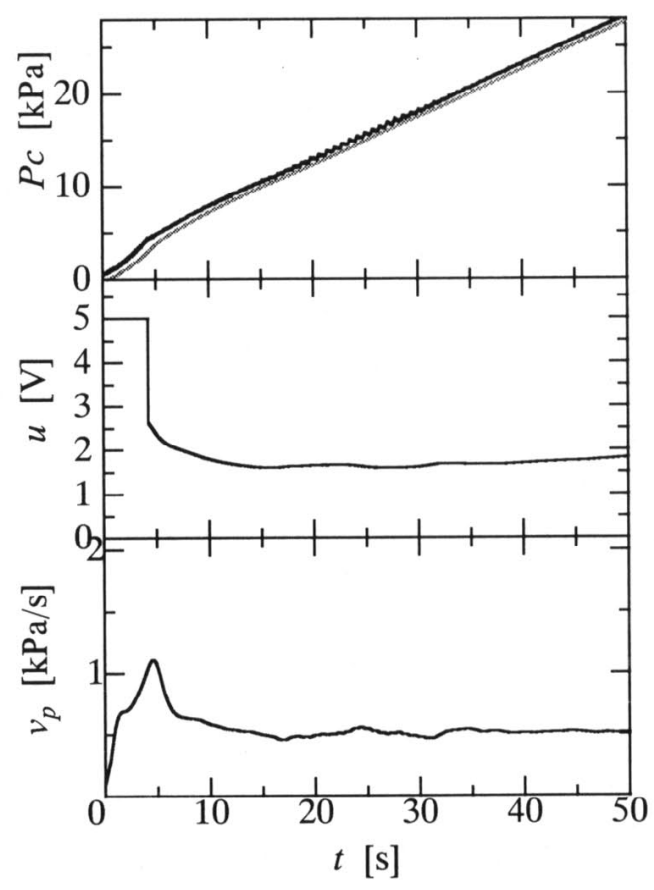

a) Pressure response and input voltage

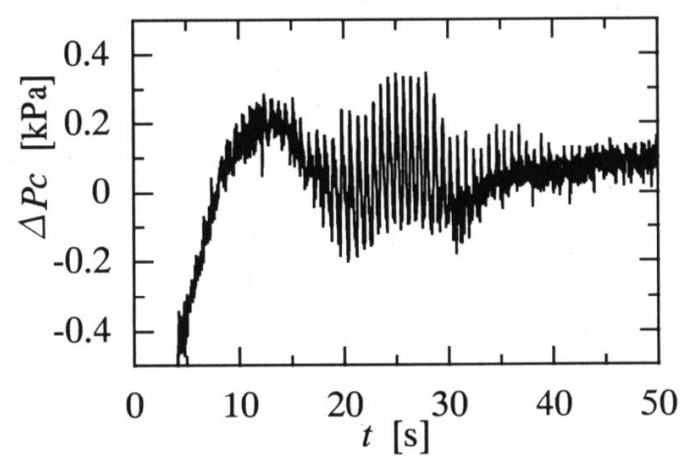

b) Mesured pressure change

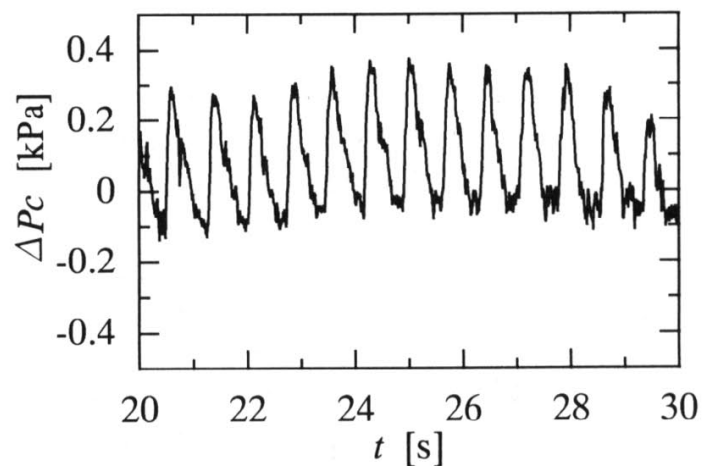

c) Enlarged view of $b$ )

Figure 15 Result of pressure control

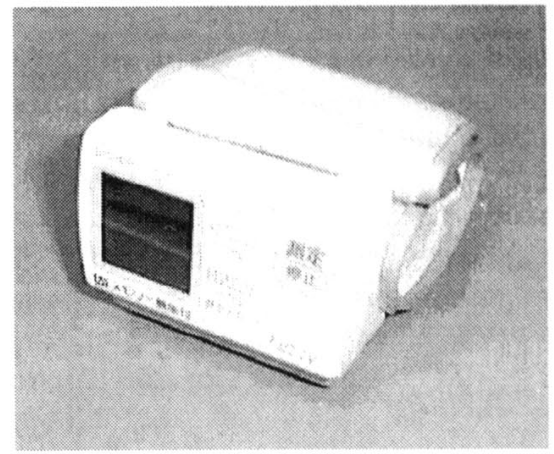

Photograph 2 Commercialized sphygmomanometer for the wrist

Photo. 2 is a sphygmomanometer commercialized. Until now, though they were the 2 pieces, it is united. It is found that the pump can control the cuff pressure in rising by the use of the appropriate control algorithm and that there is no problem on the blood pressure measurement in the pressurization.

\section{CONCLUSIONS}

It has been possible to control on the cuff pressure by controlling the pump directly. Using this, the sphygmomanometer which measure the blood pressure in pressurizing the cuff is developed. This sphygmomanometer is portable, and a measuring time is short, as the result the cooperation with the human is heightened in the blood pressure measurement.

In future following developments is desired; method compensating nonlinaerlity of the pump and the cuff, intelligent control algorithm adapting to extremely different compliance which occurs by the difference between child and adult.

\section{KNOWLAGEMENT}

In the performance of this study, it is thankful for OMRON LIFE SIENCE CORP. which offer the equipment and worthwhile discussion.

\section{REFERENCES}

1. L.A.Geddes, The Direct and Indirect Measurement of Blood Pressure, YEAR BOOK MEDICAL PUBLISHER INC., 1970

2. T.Kagawa, Pressure Control System with Proportional Valve for Non-Invasive Blood Pressure Measurement, Proceedings ,1994, pp.255-258

3. T.Kagawa, M.Shimizu, S.Ohishi, S.Ryumae, A Pressure Control System for Blood Pressure Measurement, Journal of the Japan Hydraulics \& Pneumatics Society, 1994, 24-3, pp.381-385 (in Japanese) 